\title{
REVIEW EDITOR'S STATEMENT
}

The Africa reviews editor is almost inevitably torn between wanting books reviewed as quickly as possible (which effectively means short notices, often from people within arm-twisting distance of the editorial desk) and wishing to commission and print much more detailed critical evaluations of some at least of the new literature (and sometimes some of the not-so-new literature as well). This second aim contradicts the first to the extent that in-depth reviewing takes longer to arrange (with the risk that some books may already be out of print by the time the review appears) and there is space to include fewer reviews each issue. It is important that Africa continues to cover the literature of African studies as comprehensively and promptly as possible. Nevertheless I would like to try introducing some variation into the existing format, with its concentration on medium-length notices from predominantly UK-based reviewers. In the belief that readers of Africa (and authors of books concerned) are often as well served by short but prompt notices and brief general evaluative comment as they are by less prompt, longer, but nevertheless, due to space restrictions, still far from detailed critique, I am asking for many more reviews to be limited to circa 250-500 words. This does not mean the 500-1000 word review will disappear altogether, but it is an option which will be used only for those books which by their length, complexity or significance absolutely demand such treatment. I envisage the space thus created being used in a variety of ways. I would welcome 'retrospective' review essays, looking back on important books in the light of more recent literature (Hopkin's Economic History of West Africa is one that comes to mind), or on books which for various reasons failed to receive their full critical due at the time of publication (the late John Ford's massive study of the ecology of trypanosomiasis is an example, where the significance of the argument has still to be fully appreciated by many Africanist historians and social scientists). Review articles which survey several related titles or which focus on the context as much as on the content of a given book will also be welcome. It is anticipated that major literature syntheses-some of which might from time to time be commissioned by the editor-would be handled as articles in the main part of the journal. What $I$ have in mind for the reviews section is a shorter, perhaps polemical, certainly issue-focused type of essay, capable of generating debate in which authors of reviewed books may wish to become involved. It is hoped that the reviews section of Africa will thus appear 'open', and accessible as a forum, to the widest possible spectrum of scholars, and no longer totally dependent on the 'solicited' review. This brings me to a final point. In the editorial office there is a card index of reviewers, but presently it tends to be weighted in favour of the senior, male, non-African resident, anglophone, and sociological/social anthropological sections of the academic community. I shall be happy to receive suggestions for further potential reviewers, especially those who in any way widen the scope of the present panel's composition. Having said this, however, it would be improper not to add a vote of thanks to existing reviewers, especially those who have managed to keep up with even the most anti-social of recent deadline requests. Be assured, though, that there are no plans to prune the reviewers panel, only to diversify it. 